\title{
Efeito da realidade virtual no equilíbrio postural de usuários de um centro de atenção psicossocial álcool e drogas
}

\author{
Effect of virtual reality on the postural balance of users of a \\ psychosocial care center and drugs
}

\author{
Lidiele Roque Bueno ${ }^{1}$, Nelson Francisco Serrão Junior ${ }^{1}$, Susane Graup ${ }^{1}$
}

1 - Universidade Federal do Pampa - UNIPAMPA, Uruguaiana, RS, Brasil.

lidielebueno89@gmail.com sempenho cognitivo por meio do Mini Exame do Estado Mental (utilizado como critério de inclusão), medidas antropométricas (massa corporal, estatura e circunferência da cintura), equilíbrio postural (testes Timed Up and Go, Teste de Alcance Funcional e Escala de Equilíbrio de Berg), bem como, frequência cardíaca por um aplicativo chamado Instant Heart Rate. Ainda foi aplicado um questionário visando identificar variáveis sociodemográficas. Já as informações sobre diagnóstico e terapia medicamentosa foram coletadas dos prontuários disponibilizados pelo serviço. Após a avaliação, os usuários foram submetidos à uma única sessão de até 30 minutos de jogos, para avaliar o efeito agudo da realidade virtual nestes usuários, nos momentos pré e pós intervenção, com o instrumento de intervenção virtual - Microsoft Kinect Xbox $360^{\mathrm{TM}}$. Os jogos selecionados foram: Nike Kinect Training e Kinect Adventures. Participaram do estudo 10 indivíduos de ambos os sexos com média de idade de

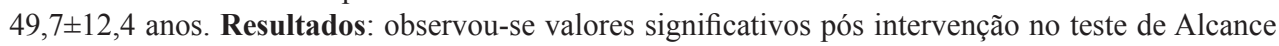

Palavras-chave: Saúde Mental; Atividade Física; Terapia Por Exercicio; Equilíbrio Postural. Funcional, no Time Up and Go e na frequência cardíaca. Optou-se por correlacionar a Escala Berg com a média obtida nos jogos de Realidade Virtual, apontando valores com diferença significativa $(\mathrm{p}=0,05)$. Conclusão: a sessão de jogos de Realidade Virtual mostrou-se importante na melhora do equilíbrio postural dos indivíduos com histórico de dependência química.

\begin{abstract}
Objective: to analyze the effects of a session of virtual reality games on the postural balance of users of a Center for Psychosocial Care Alcohol and Drugs. Method: quantitative approach aimed at evaluating patients who use a psychosocial care center in a city on the western border of Rio Grande do Sul, Brazil. Individuals underwent cognitive performance assessment through the Mini Mental State Examination (used as an inclusion criterion), anthropometric measurements (body mass, height and waist circumference), postural balance (Timed Up and Go tests, Functional Scope Test and Berg Balance Scale), as well as heart rate by an application called Instant Heart Rate. A questionnaire was used to identify sociodemographic variables. On the other hand, the information on diagnosis and drug therapy was collected from the medical records provided by the service. After the evaluation, the users were submitted to a single session of up to 30 minutes of games, to evaluate the acute effect of virtual reality in these users, in the moments before and after intervention, with the virtual intervention instrument - Microsoft Kinect Xbox 360 TM. The selected games were: Nike Kinect Training and Kinect Adventures. Ten subjects of both sexes with mean age of $49.7 \pm 12.4$ years participated in the study. Results: significant post-intervention values were observed in the Functional Reach, Time Up and Go, and heart rate test. It was chosen to correlate the Berg Scale with the average obtained in the Virtual Reality games, pointing to values with significant difference $(p=0.05)$. Conclusion: the Virtual Reality games session proved to be important in improving the postural balance of individuals with a history of chemical dependence.
\end{abstract}

Keywords:

Mental Health; Physical activity; Therapy By Exercise; Postural equilibrium. 


\section{INTRODUÇÃO}

Os Centros de Atenção Psicossocial (CAPS) são instituições especializadas em Saúde Mental, oferecendo um atendimento interdisciplinar, composto por uma equipe multiprofissional que reúne médicos, assistentes sociais, psicólogos, psiquiatras, entre outros especialistas, com objetivo de estimular sua integração cultural, social e familiar, apoiando suas iniciativas por busca de autonomia. ${ }^{1}$ Estes espaços devem funcionar como articuladores estratégicos da rede de atenção à saúde mental, promovendo vida comunitária e autonomia dos usuários. ${ }^{2}$

Em março de 2002, foram criados os Centro de Atenção Psicossocial Álcool e Drogas (CAPS Ad) que são serviços de atenção psicossocial para atendimento de pacientes com transtornos decorrentes do uso prejudicial de álcool e outras drogas, oferecendo atendimento diário, classificados como, intensivo, semi intensivo ou não intensivo. ${ }^{3}$ Esses serviços, conforme preconiza o Ministério da Saúde, devem contar com planejamento terapêutico individualizado de evolução contínua, possibilitando intervenções precoces, além de apoio de práticas de atenção comunitária e de leitos psiquiátricos em hospitais gerais. ${ }^{4}$ Vale ressaltar que a multidisciplinaridade nesses serviços é de fundamental importância para que os atendimentos possam ser mais humanizados, visando a liberdade e autonomia dos usuários.

Pensando nos indivíduos que são atendidos nestes espaços, apoiando-se na literatura, é notável que são utilizadas muitas substâncias ilícitas e lícitas, que podem causar tonturas como efeitos colaterais, influenciando negativamente as capacidades motoras e cognitivas, incluindo o tempo de reação, a coordenação e o equilíbrio. ${ }^{5}$ Neste sentido, os déficits ao nível do equilíbrio corporal podem interferir drasticamente no desempenho das atividades diárias, influenciado significativamente o bem-estar e a qualidade de vida desta população. ${ }^{6}$

O equilíbrio é a capacidade de cada ser humano de se manter ereto ou realizar movimentos de aceleração e rotação do corpo sem oscilações, desvios ou quedas. ${ }^{7}$ Para que se tenha orientação espacial estática e dinâmica, isto é, equilíbrio, dependemos de integrações que permitam ao Sistema Nervoso Central (áreas vestibulares, tronco cerebral e cerebelo) reconhecer posições e movimentos da cabeça em relação ao corpo e ao espaço, sendo que o uso de drogas, tanto com finalidade terapêutica, como exposição a substâncias químicas e tóxicas podem causar perda parcial ou total da função vestibular e/ ou coclear. ${ }^{7}$

Fatores como inatividade física, maus hábitos alimentares, tabagismo e obesidade combinados com a utilização de certos tipos de medicação, como alguns antipsicóticos, podem produzir doenças cardíacas, entre outras que afetam a condição física geral dos indivíduos e consequentemente o seu desempenho motor, sendo a prática de atividade física regular benéfica para os vários problemas de saúde física e adjuvante para a diminuição de sintomas psicológicos e de funcionamento. ${ }^{6}$

Buscando aliar a alta tecnologia à atividade física, surgem então os jogos ativos, ou exergames, nome pelo qual são conhecidas as tecnologias que exigem movimentação do corpo inteiro, combinando exercício físico com videogame. ${ }^{8}$ Essas ferramentas convertem os movimentos reais para o ambiente virtual, permitindo que os usuários se tornem ativos e pratiquem esportes virtuais, exercícios de fitness e/ ou outras atividades físicas lúdicas e interativas, por meio de movimentos que lembram as tarefas da vida real. ${ }^{8}$

Leutwyler et al. ${ }^{9,10}$ investigaram a contribuição dessa nova classe de games no âmbito da saúde mental, com a utilização do Xbox 360 com o sensor Kinect, obtendo resultados satisfatórios, como melhoria na capacidade funcional e na capacidade cognitiva dos sujeitos, além de proporcionarem uma prática prazerosa e saudável às pessoas em sofrimento psíquico.

No contexto da saúde mental, parece relevante considerar que, a partir do jogo, busca-se a possibilidade de transformação da realidade em que o jogador se encontra, fugindo da "confusão" da vida e da imperfeição do mundo para uma perfeição temporária e limitada, levando-o a uma reflexão sobre a prática do jogo. ${ }^{11}$

A utilização de tecnologias através dos jogos ativos, nos espaços do CAPS pode ser uma estratégia lúdica, que visa proporcionar promoção à saúde física e mental por meio do movimento, na forma de brincadeira ou atividade de lazer. Os jogos produzem a ideia de brincar, vencer, divertir, sem exigir a realização de movimentos altamente executados, com repetições sistematicamente computadas, sendo que momentos como este estimulam comportamentos ativos, através da atividade física de lazer.

Considerando a presença da dependência 
química e os efeitos negativos que ela origina, o estudo se justifica pela necessidade de conhecimento do perfil dos usuários do CAPS Ad na localidade do estudo, a fim de se subsidiarem futuras estratégias fundamentadas em dados referentes à realidade trabalhada, bem como se identificarem estratégias aliadas a realidade virtual (RV) voltadas a melhoria da qualidade de vida, contribuindo para o cuidado integral do indivíduo. Ainda, a partir de breve pesquisa em plataformas de publicação científica, são poucas as produções sobre a relação entre RV e o equilíbrio postural, estático e dinâmico associado ao uso de SPA. Diante destas informações, o objetivo do presente estudo foi analisar os efeitos de uma sessão de jogos de RV sobre o equilíbrio postural em usuários de um CAPS Ad da fronteira oeste do Rio Grande do Sul.

\section{MÉTODO}

Trata-se de um estudo com abordagem quantitativa que pretendeu avaliar pacientes usuários de um centro de atenção psicossocial álcool e drogas (CAPS AD) de uma cidade da fronteira oeste do Rio Grande do Sul. Este estudo foi avaliado pelo Comitê de Ética em Pesquisa da instituição dos pesquisadores e foi aprovado conforme número do parecer 2.609.301.

\section{Amostra}

A população do estudo, de acordo com as informações do CAPS AD, consiste em 28 usuários de ambos os sexos com idades entre 18 e 65 anos que frequentam diariamente os serviços. Desta forma, considerando que os paciente deveriam ter um nível cognitivo mínimo para participar da sessão de RV, para selecionar a amostra, os mesmos passaram por uma avaliação do nível cognitivo por meio do Mini Exame do Estado Mental adotando-se pontos de corte de acordo com a escolaridade: ${ }^{12,13} 17$ para os analfabetos; 22 para participantes com escolaridade entre 1 e 4 anos; 24 para os que tenham escolaridade entre 5 e 8 anos e 26 para aqueles com 9 anos ou mais anos de escolaridade. Com base nestes critérios, 19 pacientes foram classificados como elegíveis. Posteriormente, foram incluídos no estudo aqueles que aceitaram participar voluntariamente e atenderam aos seguintes critérios de inclusão: a) assinaram termo de consentimento livre e esclarecido e, b) estiveram presentes no dia das avaliações.

Após este procedimento, foram excluídos aqueles pacientes que: a) não foram capazes de realizar a marcha sem o uso de dispositivos auxiliares; b) apresentaram alterações musculoesqueléticas, visuais e/ou auditivas que impossibilitaram a realização das avaliações; c) possuíram contraindicação médica e perturbações cognitivas e/ou comportamentais que impediram a coleta de dados e; d) deixaram de participar de algum procedimento da coleta de dados ou desistissem em alguma das etapas.

\section{Procedimentos e coletas de dados}

Inicialmente realizou-se a triagem, em que o indivíduo respondia a um teste de rastreio, o Mini Exame do Estado Mental (MEEM). ${ }^{12,13}$ O MEEM é uma medida dicotômica de 30 itens, com aplicação rápida e breve, que rastreia o comprometimento das funções cognitivas. Avalia orientação temporal e espacial, memória imediata e de evocação de palavras, cálculo, nomeação, repetição, execução de um comando, leitura, escrita e habilidade visomotora. ${ }^{14}$ Para o presente estudo foi adotado o critério sugerido pela Academia Brasileira de Neurologia. ${ }^{12,13}$

Ao alcançar a pontuação mínima necessária no MEEM, o indivíduo foi considerado incluído e assinou o Termo de Consentimento Livre e Esclarecido atingindo os critérios de elegibilidade. Posteriormente o mesmo seguiu respondendo a ficha de dados socioeconômicos elaborada pelos autores.

Estas avaliações foram realizadas nas instalações do CAPS AD de forma individual, em uma sala preparada para tal. Concluindo estas etapas, foi marcado um novo dia para avaliação clínica que foi realizado em um Laboratório do curso de Fisioterapia da instituição dos pesquisadores.

No dia agendado para avaliação clínica foram realizadas avaliações antropométricas, ${ }^{15}$ testes Timed Up and Go (TUG) ${ }^{16,17}$ e de Alcance Funcional (TAF) e de Escala de Equilíbrio de Berg (EEB), ${ }^{17}$ bem como, aferida no início e final da sessão a frequência cardíaca (bpm).

O Índice de Massa Corporal (IMC) foi obtido com base no cálculo [IMC = massa corporal $(\mathrm{kg}) /$ estatura $\left.\left(\mathrm{m}^{2}\right)\right]$. Os valores de ponto de corte adotados para avaliar o estado nutricional foram propostos pela Organização Mundial da Saúde (OMS): ${ }^{18}$ baixo peso $\left(\mathrm{IMC} \leq 18,5 \mathrm{~kg} / \mathrm{m}^{2}\right)$; peso adequado $(18,5<\mathrm{IMC}<25$ $\left.\mathrm{kg} / \mathrm{m}^{2}\right)$; sobrepeso $\left(25 \leq \mathrm{IMC}<30 \mathrm{~kg} / \mathrm{m}^{2}\right)$ e obesidade $\left(\mathrm{IMC} \geq 30,00 \mathrm{~kg} / \mathrm{m}^{2}\right)$.

Para a avaliação antropométrica foram aferidos massa corporal atual, estatura e circunferência de cintura seguindo as técnicas propostas por Callaway, ${ }^{15}$ 
para os grupos etários estudados. Para a avaliação da massa corporal, fez-se uso de uma balança antropométrica mecânica com capacidade de $150 \mathrm{~kg}$ com precisão de $100 \mathrm{~g}$ e para a estatura foi utilizada uma régua antropométrica com escala de $2,00 \mathrm{~m}$ em alumínio anodizado, com escala de $0,5 \mathrm{~cm}$ ambas da marca 110-CH Welmy, seguindo os passos descritos na literatura. ${ }^{15}$

A circunferência da cintura foi aferida com fita métrica simples e inelástica, onde foi solicitado que o participante estivesse em posição ereta, com abdômen levemente relaxado, braços lateralmente ao corpo e com os pés unidos. A obesidade abdominal foi determinada de acordo com os valores de circunferência abdominal $\geq 102 \mathrm{~cm}$ para homens e $\geq 88$ para mulheres. ${ }^{15}$

Para avaliação do equilíbrio estático e dinâmico, os indivíduos foram submetidos a EEB, TUG e ao TAF. A EEB ${ }^{17}$ trata-se de um instrumento padronizado e de fácil aplicação que avalia o equilíbrio do indivíduo em 14 situações representativas da funcionalidade no ambiente diário, tais como: ficar em pé, levantar-se, andar, inclinar-se a frente, transferir-se, virar-se, entre outras. ${ }^{17}$ A pontuação máxima a ser alcançada é de 56 pontos, e cada item possui uma escala ordinal de cinco alternativas, assim, a escala varia de zero (incapacidade de realizar a tarefa) a quatro (capacidade de realizar a tarefa sem dificuldade) pontos. Os pontos são subtraídos caso o tempo ou a distância para completar uma tarefa não sejam atingidos ou o indivíduo necessite de supervisão para execução da tarefa. A avaliação é descrita como simples, fácil de ser aplicada e segura. ${ }^{17}$ De acordo com a literatura, quanto menor a pontuação atingida pelo indivíduo, maior será a possibilidade de queda.

O TUG, é um teste de levantar e caminhar cronometrado que avalia o nível de mobilidade funcional de forma rápida e prática, ${ }^{16,17}$ e consiste em mensurar em segundos o tempo gasto por um indivíduo para levantar de uma cadeira, andar uma distância de 3 metros, dar a volta, retornar a cadeira e sentar novamente. Para a realização do teste foram utilizados uma cadeira de braços, um cronômetro e uma ficha de anotação dos dados, sendo que o assento da cadeira deve possuir $46 \mathrm{~cm}$ de altura. ${ }^{16,17}$ Os pontos de corte são: até 10 segundos - normal para adultos e indica um baixo risco de quedas; entre 11 a 20 segundos - médio risco de quedas e independência parcial; maior que 20 segundos - alto risco de quedas e déficit importante da mobilidade física. ${ }^{17}$
O TAF seguiu os padrões de Duncan, ${ }^{19}$ com os pés paralelos em posição confortável, em flexão de ombro a $90^{\circ}$, realizando um movimento de deslocamento anterior de tronco, simulando uma atividade extremamente funcional, e não sendo permitido rodar o tronco e nem retirar os pés do chão. Utilizou-se fita métrica para mensuração da distância do membro superior. O teste foi executado três vezes, obtendo-se a média dos três valores, sendo considerado valor normal de $\geq 14 \mathrm{~cm}$ e pontuações inferiores caracterizam maior risco de quedas. ${ }^{19}$

Para a avaliação da frequência cardíaca, foi utilizado um aplicativo chamado Instant Heart Rate, para seu funcionamento utilizou-se a câmera e a luz do flash de um smartphone com android para medir os batimentos cardíacos, onde foi posicionado o dedo indicador esquerdo do indivíduo sobre a lente da câmera, pressionando-o por 15 segundos, pré e pós intervenção, assim o aplicativo detectou suaves mudanças de coloração da pele, causadas pelo fluxo de sangue entre cada batida do coração e os valores fornecidos pelo aplicativo foram anotadas em um planilha.

Após avaliados, foram submetidos à uma única sessão, individualmente, a fim de avaliar o efeito agudo da RV sobre estes indivíduos, com duração de até 30 minutos, utilizando-se o instrumento de intervenção virtual - console Microsoft Kinect Xbox 360 TM, o qual possui sensores capazes de captar os movimentos do jogador, sem a necessidade de uma interface entre o mesmo e a máquina. Os jogos selecionados foram: Nike Kinect Training (com duração de cinco minutos) e Kinect Adventures (River Rush - dois minutos, Reflex Ridge - um minuto e trinta segundos, e 20.000 Leaks - dois minutos e trinta segundos. Entre um jogo e o próximo, foi dado um descanso de três minutos. A escolha dos jogos ocorreu por englobarem habilidades motoras básicas, como: baixar, saltar, levantar, baixar e levantar os braços e inclinar o tronco.

\section{Intervenção com a realidade virtual}

Os jogos selecionados promovem ajustes posturais necessários para o alcance de objetos, deslocamento multidirecional do corpo e troca de passos. O Nike Kinect Training conta com um personal trainer virtual e exercícios para melhorar o condicionamento físico, proporcionando movimentação de membros inferiores e superiores em alta intensidade por no máximo 5 minutos. $\mathrm{O}$ jogo avalia como o participante mexe seu corpo, 
força física e capacidade atlética, identificando áreas e pontos onde o participante deve melhorar e, assim, cria uma série de exercícios personalizados para cada um; ele corrige ainda se o mesmo está fazendo os movimentos de forma errônea, onde este jogo foi utilizado para o aquecimento inicial. No River Rush o avatar ("boneco" virtual que simula o indivíduo) está sobre uma jangada que deve ser controlada por deslocamentos do centro de gravidade do jogador com intuito de tocar em esferas virtuais no leito de um rio. No jogo Reflex Ridge o avatar se mantém sobre uma plataforma que se move sobre trilhos, devendo desviar de obstáculos e tocar em esferas virtuais. E no 20.000 Leaks o avatar do jogador está dentro de um cubo de vidro sob a água. Os jogadores têm que movimentar partes do corpo para tampar buracos no cubo, evitando vazamentos. Ao final de cada partida (River Rush, Reflex Ridge e 20.000 Leaks) foi computado o escore gerado pelo jogo e anotado em uma planilha.

Com o intuito de verificar o efeito do programa de RV com videogame ativo (VGA), realizou-se as seguintes avaliações: MEEM, EEB, TUG e TAF nos momentos pré e pós intervenção. Os dados foram coletados nos meses de outubro e novembro de 2018 .

\section{Análise estatística}

Os resultados obtidos foram expressos, descritivamente, em média, desvio padrão e porcentagem. Para comparar as diferentes variáveis pré e pós intervenção foi realizada o teste $\mathrm{t}$ pareado. As correlações relevantes foram investigadas empregando-se os coeficientes de correlação de Pearson. A análise inferencial foi realizada através do programa estatístico Graph Pad Prisma, versão 6.0. Em todas as análises foi considerado o nível de significância de $5 \%(p<0,05)$ e intervalo de confiança de $95 \%$.

\section{RESULTADOS}

A amostra do estudo foi constituída por 10 indivíduos $(\mathrm{n}=10)$ com média de idade de 49,7 712,4 anos e tempo de uso de álcool e/ou drogas 33,6 $2 \pm 13,8$ anos (Tabela 1).

Tabela 1 - Valores descritivos das variáveis dos usuários do CAPS Ad, 2018.

\begin{tabular}{lcc}
\hline VARIÁVEIS & Média & Desvio padrão \\
\hline Idade (anos) & 49,7 & 12,4 \\
Massa corporal $(\mathrm{kg})$ & 71,1 & 14,15 \\
Estatura (m) & 1,65 & 0,08 \\
Índice de Massa corporal $\left(\mathrm{kg} / \mathrm{m}^{2}\right)$ & 25,7 & 3,08 \\
Circunferência da cintura (cm) & 90,9 & 13,40 \\
Tempo de uso de álcool e/ou drogas (anos) & 33,6 & 13,8 \\
Realidade Virtual (escore) & 78,5 & 25,28 \\
\hline
\end{tabular}

Legenda: $\mathrm{n}=$ número amostral; $\mathrm{kg}=$ quilogramas; $\mathrm{m}$-=metros; $\mathrm{cm}=$ =entímetro

A Tabela 2, apresenta a distribuição de frequência das variáveis analisadas nos usuários do CAPS Ad avaliados, sendo possível identificar que a maioria era do sexo masculino $(90 \%)$, na faixa etária acima de 40 anos de idade (80\%), solteiro $(60 \%)$ e com nível fundamental incompleto $(60 \%)$.

Em relação ao uso de substâncias (Tabela 2), a maioria fazia uso de tabaco $(70 \%)$ e álcool $(60 \%)$ e apresentava CID F 19 - Transtornos mentais e comportamentais devido ao uso de múltiplas drogas e ao uso de outras substancias psicoativas (70\%). Considerando o tratamento terapêutico, todos os usuários faziam polimedicação, usando antipsicóticos e anticonvulsivantes.

O risco cardiovascular dos indivíduos deste estudo foi avaliado pela circunferência abdominal, que apresentou média de 90,92 $\pm 13,40$, estando dentro dos parâmetros ${ }^{(15)} \geq 102$, visto que a amostra do estudo é composta majoritariamente pelo sexo masculino, onde esta média não foi caracterizada como risco aumentado para doenças cardiovasculares. Vale ressaltar que $70 \%$ dos usuários estavam dentro dos parâmetros e 30\% apresentaram risco cardiovascular elevado (para homens $\geq 102$ e para mulheres $\geq 88$ ). 
Tabela 2 - Frequências absolutas e relativas das variáveis analisadas nos usuários do Caps Ad, 2018.

\begin{tabular}{|c|c|c|}
\hline VARIÁVEIS & $\mathbf{N}$ & $\%$ \\
\hline \multicolumn{3}{|l|}{ Sexo } \\
\hline Masculino & 9 & 90 \\
\hline Feminino & 1 & 10 \\
\hline \multicolumn{3}{|l|}{ Faixa etária } \\
\hline Até 40 anos & 2 & 20 \\
\hline Mais de 40 anos & 8 & 80 \\
\hline \multicolumn{3}{|l|}{ Classificação do IMC } \\
\hline Baixo peso & 1 & 10 \\
\hline Eutróficos & 4 & 40 \\
\hline Sobrepeso & 4 & 40 \\
\hline Obesidade & 1 & 10 \\
\hline \multicolumn{3}{|l|}{ Classificação do RCQ } \\
\hline Normal & 7 & 70 \\
\hline Risco aumentado & 3 & 30 \\
\hline \multicolumn{3}{|l|}{ Estado civil } \\
\hline Solteiro & 6 & 60 \\
\hline Casado & 1 & 10 \\
\hline Separado & 3 & 30 \\
\hline \multicolumn{3}{|l|}{ Escolaridade } \\
\hline Fundamental incompleto & 6 & 60 \\
\hline Médio completo & 4 & 40 \\
\hline \multicolumn{3}{|l|}{ Ocupação } \\
\hline Aposentado & 2 & 20 \\
\hline Desempregado & 3 & 30 \\
\hline Outro & 5 & 50 \\
\hline \multicolumn{3}{|l|}{ Tipo de auxílio financeiro recebido } \\
\hline Benefício de Prestação Continuada (BPC) & 8 & 80 \\
\hline Bolsa Família & 1 & 10 \\
\hline Nenhum & 1 & 10 \\
\hline \multicolumn{3}{|l|}{ Consumo de substância } \\
\hline Álcool & 6 & 60 \\
\hline Cannabis & 1 & 10 \\
\hline Katovit/Flakka & 1 & 10 \\
\hline Cannabis e álcool & 1 & 10 \\
\hline Cannabis e cocaína & 1 & 10 \\
\hline Tabaco & 7 & 70 \\
\hline \multicolumn{3}{|l|}{ Diagnóstico } \\
\hline CID-10 Primário & 4 & 40 \\
\hline CID-10 Secundário & 6 & 60 \\
\hline F 10 - Transtornos mentais e comportamentais devido ao uso de álcool & 4 & 40 \\
\hline $\begin{array}{l}\text { F } 19 \text { - Transtornos mentais e comportamentais devido ao uso de múltiplas drogas e ao uso de outras } \\
\text { substancias psicoativas }\end{array}$ & 7 & 70 \\
\hline F 20.1 - Esquizofrenia paranoide & 1 & 10 \\
\hline F 25.1 - Transtorno esquizoafetivo do tipo depressivo & 1 & 10 \\
\hline F 29.0 - Psicose não-orgânica não especificada & 1 & 10 \\
\hline F 31.5 - Transtorno afetivo bipolar, episódio atual depressivo grave com sintomas psicóticos & 1 & 10 \\
\hline F 32.2 - Episódio depressivo grave sem sintomas psicóticos & 1 & 10 \\
\hline F 70.0 - Retardo mental leve & 1 & 10 \\
\hline \multicolumn{3}{|l|}{ Terapia medicamentosa } \\
\hline Antipsicóticos & 10 & 100 \\
\hline Antidepressivos & 4 & 40 \\
\hline Anticonvulsivantes & 10 & 100 \\
\hline Antiparkinsoniano & 3 & 30 \\
\hline
\end{tabular}

Legenda: CID-10: Classificação Internacional de Doenças 
Sobre as variáveis de equilíbrio estático e dinâmico, é possível observar na Tabela 3 que houve diferença significativa no Teste de alcance funcional $(\mathrm{p}=0,003)$, no Teste timed up and $\mathrm{go}(\mathrm{p}=0,005)$ entre $\mathrm{o}$ pré e o pós teste, sendo que após a sessão de realidade virtual, os resultados dos testes foram melhores.
A Figura 1 evidencia que os valores da Escala de Equilíbrio de Berg do pós teste tiveram correlação moderada $(\mathrm{r}=0,63)$, diretamente proporcional, com os valores médios dos escores nos jogos de realidade virtual, indicando que quanto maior o equilíbrio maiores os escores nos jogos.

Tabela 3 - Características da amostra em relação aos valores pré e pós intervenção.

\begin{tabular}{l|c|c|c}
\hline \multicolumn{1}{c|}{ Variáveis } & Pré-Intervenção & Pós-Intervenção & P \\
Escala de Berg, pontos & $52,5 \pm 3,9$ & $53,2 \pm 3,7$ & 0,17 \\
Teste de alcance funcional, cm & $25,9 \pm 8,6$ & $30,1 \pm 7,3$ & $0,003^{*}$ \\
Teste timed up and go, segundos & $12,5 \pm 2,1$ & $10,8 \pm 2,2$ & $0,005^{*}$ \\
Frequência cardíaca, bpm & $70,6 \pm 15,5$ & $82,7 \pm 19,2$ & $0,005^{*}$ \\
\hline
\end{tabular}

Legenda: SF-36: Short Form 36; cm: centímetros; bpm: batimentos por minutos. P: valor de $\mathrm{p}(\mathrm{p}<0,05) ;\left({ }^{*}\right)$ : Valores estatísticamente significantes $(\mathrm{p}<0,05)$.

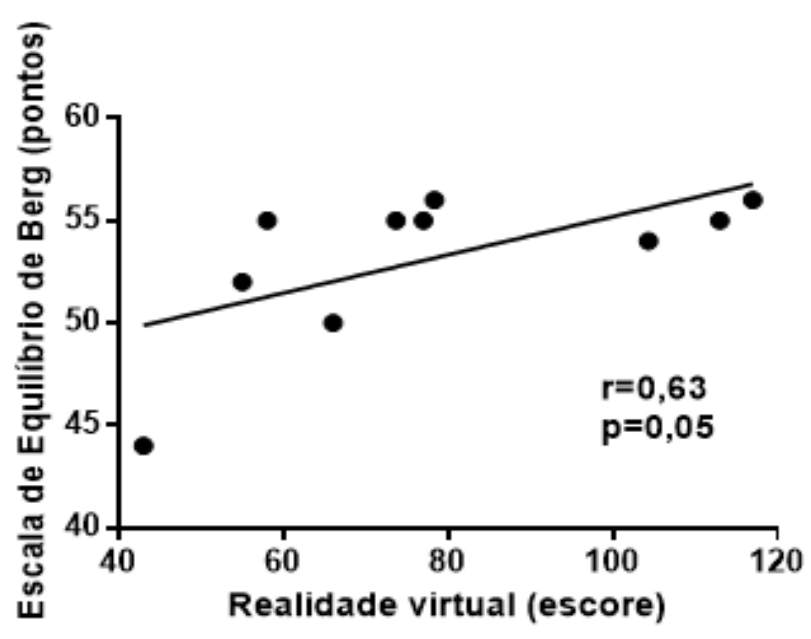

Figura 1 - Correlação entre a pontuação da média de jogos de Realidade Virtual e pontuação pós intervenção da Escala de Equilíbrio de Berg.

\section{DISCUSSÃO}

Com este estudo foi possível identificar uma dominância de usuários do sexo masculino, resultado semelhante ao estudo realizado por Oliveira et al., ${ }^{20}$ que também encontrou a maioria de indivíduos do sexo masculino em um CAPS Ad do Ceará, em outro estudo $^{21}$ revelou que os homens, em sua maioria, estão mais propensos a usar drogas ilícitas mais precocemente, por mais tempo e com uma frequência maior que as mulheres.

De Oliveira et al. ${ }^{22}$ refere em seu estudo que embora a literatura internacional registre um aumento progressivo no consumo de substâncias psicoativas nas últimas décadas por mulheres, outros estudos ${ }^{23}$ apontam que elas buscam e permanecem menos no tratamento para transtornos decorrentes do consumo de substâncias psicoativas em decorrência dos aspectos históricos e culturais que envolvem o papel da mulher na sociedade, do estigma social relacionado ao consumo de substâncias psicoativas e da alteração da autoimagem. Estes aspectos reprimem as suas atitudes, favorecendo o sentimento de vergonha e retardando assim a busca de tratamento para suas questões de dependência química. ${ }^{23,24}$

Os diagnósticos de transtornos mentais e comportamentaismais frequentes entreos participantes do estudo estão apresentados na Tabela 2. Observa-se 
que $70 \%$ dos participantes apresentaram o diagnóstico de transtornos mentais/comportamentais devidos ao uso de múltiplas drogas psicoativas (transtorno psicótico) seguido por $40 \%$ dos participantes com transtornos mentais e comportamentais devido ao uso de álcool. Um dado relevante refere-se à presença de comorbidades psiquiátricas. Dos 10 participantes, 6 (60\%) apresentaram outro diagnóstico psiquiátrico concomitante, como por exemplo, esquizofrenia paranoide, entre outros.

Cabe destacar que uma parcela significativa dos participantes apresentou outras comorbidades psiquiátricas associadas aos transtornos mentais e comportamentais devido ao uso de múltiplas substâncias psicoativas, bem como relacionados ao álcool. Nesse sentido, é importante destacar que o duplo diagnóstico geralmente se desenvolve mais tardiamente e interfere na adesão ao tratamento, além de haver a possibilidade de comportamentos de risco e ideação suicida. ${ }^{25}$ As comorbidades psiquiátricas podem interferir na identificação do uso abusivo de substâncias como no outro transtorno mental e viceversa.

O estado civil e o nível de escolaridade também foram pesquisados, sendo que a maioria era solteiro, similar aos dados apresentados por Andolfatto et al., ${ }^{26}$ em que percebeu-se um número elevado de pessoas que não apresentam relacionamento estável, vivendo com cônjuges e filhos, refletindo um dos problemas do uso de drogas, que é o isolamento e a dificuldade de fixar um relacionamento.

Relacionado ao nível de escolaridade, a maior porcentagem dos indivíduos possui ensino fundamental incompleto e com tempo de dependência em média de 33,6 $6 \pm 13,8$ anos, sendo similar aos resultados apresentados por Malagodi et al. ${ }^{27}$ em sua pesquisa, em que a baixa escolaridade era maior e o tempo de dependência superior a 10 anos. Segundo a OMS, ${ }^{28}$ o início do consumo de álcool, cigarro e outras drogas ocorrem predominantemente durante a adolescência, o que possivelmente justifica o fato de a maior parte dos indivíduos consumirem o álcool e outras substâncias por um longo período antes de buscarem tratamento. Ainda, como em muitos casos, o início da dependência é precoce, o que geralmente acarreta prejuízos cognitivos de percepções, memória e pensamentos, resultando em déficit de aprendizagem, de rendimento e no consequente abandono escolar em idades mais jovens, o que explicaria a baixa escolaridade observada em nossa amostra.
Em relação às substâncias de preferência dos usuários do CAPS Ad, pode-se observar que o álcool e o tabaco possuíam maior índice de usuários. Outras drogas também estão entre as preferidas dos usuários, como cannabis, cocaína e katovit/flakka (injetável). Alguns indivíduos apresentam consumo de mais do que uma substância, como se pode analisar na Tabela 2. A predominância do uso de álcool também está presente no estudo de Silva, ${ }^{29}$ em que $79,6 \%$ dos usuários utilizavam essa substância. No que se refere à medicação todos os participantes tinham prescrição de mais de um fármaco, sendo predominante os psicofármacos do grupo de antipsicóticos e anticonvulsivantes.

Durante o período de tratamento são relatados em outros estudos ${ }^{30}$ que os usuários apresentam agressividade, fissura pela droga, humor instável, perda de apetite, insônia e hipersexualidade. De acordo com Jesus et al. $^{30}$ não existe uma abordagem terapêutica farmacológica que finalize verdadeiramente os sintomas como inquietação, insônia e aspectos depressivos. Existe sim uma intervenção farmacológica que pode ser utilizada para atenuar a intensidade da síndrome de abstinência (sintomas mentais e físicos que ocorrem após a interrupção ou diminuição no consumo de uma substancia que causa dependência).

Quanto ao tempo de consumo de drogas e álcool ao longo da vida por indivíduos em situação de dependência química, algumas pesquisas têm reportado que esta variável, juntamente com a quantidade total da substância consumida, possui relação direta com a degeneração cerebelar, sendo que o maior tempo de exposição prejudicaria de maneira progressiva a manutenção da postura e do equilíbrio corporal. $^{27}$ No entanto, não foram encontrados estudos que analisassem esta relação envolvendo outras variáveis da aptidão física em indivíduos nesta condição.

Com relação aos dados do IMC os indivíduos do CAPS Ad, a metade apresentou excesso de peso (sobrepeso+obesidade). Em estudo semelhante de Willhelm, Escobar e Perry, ${ }^{31}$ detectaram maior porcentagem de indivíduos eutróficos entre dependentes de crack internados em uma unidade de tratamento de dependência química. Ainda, segundo os mesmos autores,${ }^{31}$ deve-se atentar para a possibilidade de aumento acentuado da massa corporal desses indivíduos ao longo do tratamento, uma vez que estes em geral chegam à internação muito debilitados 
e, por conta da abstinência, podem desenvolver um mecanismo de hiperfagia rebote. Esse mecanismo funcionaria para restabelecer os neurotransmissores (dopamina e serotonina, principalmente) do sistema de recompensa, o que pode gerar abrupto aumento da massa corporal e consequente estado de sobrepeso e obesidade, conforme encontrado em $50 \%$ de nossa amostra e corroborando aos achados de Willhelm, Escobar e Perry. ${ }^{31}$

Neste estudo relacionado ao risco cardiovascular, $70 \%$ dos usuários estão dentro dos parâmetros recomendados e $30 \%$ apresentaram risco cardiovascular elevado, sendo para homens $\geq 102$ e para mulheres $\geq 88$. Recine e Radaelli ${ }^{(32)}$ indicam que homens e mulheres com gordura corporal relativa acima dos valores aceitáveis de referência, apresentam maiores riscos para doenças crônicas não transmissíveis como cardiopatias, acidentes vasculares cerebrais, hipertensão, dislipidemias, diabetes mellitus, aterosclerose, cálculo biliar, neoplasias, entre outras.

Houve melhoras pós intervenção quando comparada à avaliação pré intervenção nos testes motores de Alcance Funcional $(p<0,003)$ com importante redução de tempo entre pré e pós intervenção no teste TUG $(p<0,005)$. Os resultados do desempenho do TAF demonstraram, após o programa de treino (pós-teste), diferenças estatisticamente significantes. O equilíbrio e o controle postural foram aspectos que melhoraram após a aplicação dos jogos de RV. Com relação ao TUG, nosso estudo corrobora aos achados de Santos et al., ${ }^{33}$ no qual o grupo que realizou o treinamento com Xbox 360 associado ao Kinect ${ }^{\mathrm{TM}}$, melhorou a mobilidade, capacidade funcional e redução de incidência a queda em idosos, justificando assim, o uso de jogos de RV para melhoria da condição do equilíbrio postural também desta população alvo do estudo, demonstrando ainda que os indivíduos diminuíram o tempo de execução comparado ao pré-teste.

Em relação aos dados da $\mathrm{FC}$ expressos na Tabela 3, observou-se que em apenas uma sessão de $\mathrm{RV}$, a mesma proporcionou aumento significativo $(\mathrm{p}=0,005)$ da $\mathrm{FC}$, quando comparado ao momento pré intervenção $(70,6 \pm 15,5$ versus $82,7 \pm 19,2)$. Tal característica pode ter sido observada pela maior execução de movimentos, corroborando aos resultados obtidos no estudo de Junior, ${ }^{34}$ onde analisou o efeito agudo da acupuntura, atividade física lúdica e da RV sobre a dor em pacientes cardíacos.
Em relação aos resultados obtidos nos testes de equilíbrio, não foram identificadas diferenças estatisticamente significantes nos dados relacionados à EEB ( $p=0,17)$; assim como o estudo de Pelisser ${ }^{35}$ sobre correlação entre os resultados obtidos em testes motores e o desempenho em exergames em idosos. Embora a utilização da EEB seja largamente utilizada no meio científico, ${ }^{36,37}$ diferenças entre a capacidade motora da amostra investigada e da população utilizada, no estudo de Myiamoto $^{38}$ para a adaptação da escala no Brasil, pode ter sido determinante para os resultados não demonstrarem diferenças entre os grupos no presente estudo.

Porém, no presente estudo, também ficou evidenciado correlação moderada $(\mathrm{r}=0,63, \mathrm{p}=0,05)$ entre os resultados da RV e as variáveis de equilíbrio a partir da EEB (Figura 2), apontando valores com diferença significativa $(p=0,05)$, demonstrando que quanto melhor o desempenho frente aos jogos de RV melhor a condição relacionada ao equilíbrio estático e dinâmico. Estudos mais recentes ${ }^{39,40}$ sugerem a utilização dos jogos de RV na elaboração de protocolos de treinamento de curto, médio e longo prazo, sendo que resultados satisfatórios em relação à melhora do desempenho motor da população idosa já foram identificados. Assim como os resultados satisfatórios apresentados em outras pesquisas, a população do nosso estudo também apresenta resultados positivos, (como por exemplo, melhora na distância mensurada de membro superior no TAF, no escore mensurado na EEB), reforçando que a utilização de jogos de RV são benéficos para a condição do equilíbrio postural.

Nosso estudo apresentou como limitação um número de usuários reduzido, devido aos critérios de inclusão descritos. Ainda assim, os dados aqui levantados são de grande relevância e interesse para os profissionais de saúde que atuam com pessoas dependentes de substâncias psicoativas, especialmente pela escassez de dados disponíveis sobre variáveis relacionadas à esta população, podendo assim oferecer subsídios iniciais para ações que envolvam a implementação de programas de exercícios físicos associadas a Realidade Virtual como estratégia terapêutica, sendo esta de aspecto inovador, baixo custo e com excelentes resultados à curto prazo.

No entanto, a literatura pouco referencia estudos relacionados à $\mathrm{RV}$ e usuários dependentes químicos, uma vez que há maiores publicações relacionadas a outras temáticas, tais como, acidente vascular encefálico, paralisia cerebral, doença de 
Parkinson, entre outras. Portanto a dificuldade de comparar os resultados encontrados no estudo em questão, apesar de ser uma intervenção que propõe melhorar o déficit de equilíbrio em adultos, mas é necessário que sejam realizados e publicados novos estudos.

\section{CONCLUSÃO}

Verificou-se que houve efeito imediato positivo da Realidade Virtual no equilíbrio postural dos indivíduos quando avaliados pelo Teste de Alcance Funcional e Escala de Equilíbrio de Berg.

\section{Agradecimentos}

\section{Agradecemos aos usuários do Centro de Atenção Psicossocial Álcool e Drogas pela adesão à proposta de Realidade Virtual.}

\section{REFERÊNCIAS}

1. Brasil. Ministério da Saúde. Secretaria de Atenção à Saúde. Departamento de Ações Programáticas Estratégicas. Saúde mental no SUS: Os Centros de Atenção Psicossocial. Brasília: Ministério da Saúde, 2004. Disponível em: <http://www.ccs. saude.gov.br/saude_mental/pdf/sm_sus.pdf $>$.

2. Larentis CP, Maggi A. Centros de Atenção Psicossocial Álcool e Drogas e a Psicologia. Aletheia, 2012;37:121-32. Disponível em: $\quad<$ http://pepsic.bvsalud.org/scielo.php?script=sci abstract\&pid=S1413-03942012000100009>.

3. Alverga AR, Dimenstein M. A reforma psiquiátrica e os desafios na desinstitucionalização da loucura. Interface Comunicação, Saúde, Educação, 2006. doi: http://dx.doi. Org/0.1590/S1414-32832006000200003.

4. Brasil. Ministério da Saúde. Secretaria de Atenção à Saúde. DAPE. Coordenação Geral de Saúde Mental. Reforma psiquiátrica e política de saúde mental no Brasil. Documento apresentado à Conferência Regional de Reforma dos Serviços de Saúde Mental : 15 anos depois de Caracas. OPAS. Brasília, 2005. Disponível em: <http://bvsms.saude.gov.br/bvs/publicacoes/ Relatorio15 anos_Caracas.pdf>.

5. Schmidt PMS, Giordani AM, Rossi AG, Coser PL. Avaliação do equilíbrio em alcoólicos. Braz J Otorhinolaryngol, 2010. V.76(2), 148-155. doi: http://dx.doi.org/10.1590/S180886942010000200002

6. Lopes MVF. Efeitos de um programa de atividade física com recurso a exergaming em indivíduos com perturbação pela utilização de substâncias e doença mental severa em tratamento numa comunidade terapêutica [tese]. Rio de Janeiro: Escola Superior de Tecnologia da Saúde do Instituto Politécnico do Porto; 2015. Disponível em: <http://recipp.ipp. pt/handle/10400.22/7869>.

7. Giordani AM. A influência do alcoolismo no equilíbrio postural [tese]. Rio Grande do Sul: UFSM; 2004. Disponível em: $\quad<$ https://repositorio.ufsm.br/bitstream/handle/1/6413/ ALINE\%20GIORDANI.pdf? sequence=1\&isAllowed $=\mathrm{y}>$.

8. Medeiros PD, Capistranoa R, Zequinão MA, Silva SAD, Beltrame TS, Cardoso FL. Exergames como ferramenta de aquisiçãoedesenvolvimentodehabilidadesecapacidadesmotoras: uma revisão sistemática. Rev Paul Pediatr 2017; 35(4):464-71. doi: $\quad$ http://dx.doi.org/10.1590/1984-0462/;2017;35;4;00013 9. Leutwyler H, Hubbard EM, Vinogradov S, Dowling GA. Videogames to Promote Physical Activity in Older Adults with Schizophrenia. Games Health J 2012;1(5):381-383. doi: http:// dx.doi.org/10.1089/g4h.2012.0051

10. Leutwyler H, Hubbard EM, Dowling GA. Adherence to a Videogame-Based Physical Activity Program for Older Adults with Schizophrenia. Games Health J 2014;3(4):227-33. doi: http://dx.doi.org/10.1089/g4h.2014.0006

11. Huizinga J. Homo ludens: o jogo como elemento da cultura. 8 ed. São Paulo: Perspectiva, 2014.

12. Melo DM, Barbosa AJG, Neri AL. Mini Exame do Estado Mental: evidências de validade baseadas na estrutura interna. Avaliação Psicológica, 2017;16(2):161-8. doi: http://dx.doi. org/10.15689/AP.2017.1602.06

13. Belintani DC, Bueno DRS, Fattori A, Guariento ME. Funcionalidade de idosos não portadores de demência atendidos em serviço de referência. Revista Brasileira de Neurologia e Psiquiatria 2017;21(1):6-16.

14. Alfieri FM, Teodori RM, Montebelo MIL. Mobilidade funcional de idosos submetidos a intervenção fisioterapêutica. Saúde Rev 2004;6(14):45-50.

15. Callaway CW, Chumlea WC, Bouchard C, Himes JH, Lohman TG, Martin AD. Circumferences. In: Lohman TG, Roche AF, Martorell R, Editors. Anthropometric standardization reference manual. Chapaing: Human Kinetics Books; p.39-54. 1988.

16. Podsiadlo D, Richardson S. The timed "Up \& Go": a test of basic functional mobility for frail elderly pearsons. J Am Geriatr Soc 1991; 39(2):142-8.

17. Sousa N, Marques U. Prevenção da queda do idoso. As alterações induzidas pelo treino da força no desempenho do Timed Get-Up \& Go Test e do Functional Reach Test. Revista Digital. 2002;8(53):1-3.

18. World Health Organization (WHO). Physical status: the use and interpretation of antropometry. Report of a WHO Expert Commitee. WHO Technical Report Series, 854 Geneve: WHO; 1995. Disponível em: <http://whqlibdoc.who.int/trs/WHO TRS_854.pdf?ua $=1>$.

19. Duncan PW, Weiner DK, Chandler J, Studenski S. Functional reach: a new clinical measure of balance. J Gerontology. 1990; 45:192-97.

20. Oliveira EN, Silva MWP, Eloia SC, Mororó FWP, Lima GF, Matias MMM. Caracterização da clientela atendida em centro de atenção psicossocial - álcool e drogas. Revista da Rede de Enfermagem do Nordeste 2013;14(4):748-56.

21. Gossop M, Manning V, Ridge G. Concurrent use and order of use of cocaine and alcohol: behavioural differences between users of crack cocaine and cocaine powder. Adiction. 2006;101(9):1292-8. doi: http://dx.doi.org/10.1111/j.13600443.2006.01497.x

22. De Oliveira VC, Capistrano FC, Ferreira ACZ, Kalinke LP, Felix JVC, Maftum MA. Perfil sociodemográfico e clínico de pessoas atendidas em um caps ad do sul do brasil. Rev Baiana 
Enferm 2017;31(1):e16350. doi: http://dx.doi.org/10.18471/rbe v31i1.16350

23. Peixoto C, Prado CHO, Rodrigues CP, Cheda JND, Mota LBT, Veras AB. Impacto do perfil clínico e sociodemográfico na adesão ao tratamento de pacientes de um Centro de Atenção Psicossocial a Usuários de Álcool e Drogas (CAPS AD). J bras psiquiatr 2010;59(4):317-21. doi: http://dx.doi.org/10.1590/ S0047-20852010000400008

24. Greenfield SF, Back SE, Lawson K, Brady KT. Substance abuse in women. Psychiatr Clin North Am. 2010;33(2):339-55. doi: http://dx.doi.org/10.1016/j.psc.2010.01.004

25. Langas AM, Ulrik FM, Stein O. Comorbid mental disorders in substance users from a single catchment area - a clinical study. BMC Psychiatry 2011;11:25. doi: http://dx.doi. org/10.1186/1471-244X-11-25

26. Andolfatto I, Frighetto M, Winck DR, Dambrós BP. Caracterização de usuários de álcool e drogas atendidos pelo centro de atenção psicossocial (CAPS-I) de um município do meio oeste catarinense. Unoesc \& Ciência-ACBS 2016;7(1):3138.

27. Malagodi BM, Greguol M, Serassuelo Junior H. Analise do equilíbrio corporal e aptidão física de indivíduos em tratamento para dependência química. Revista Brasileira de atividade Física e Saúde 2018;23:e0012. doi: http://dx.doi.org/10.12820/ rbafs. $23 \mathrm{e} 0012$

28. Organização Mundial da Saúde - World Health Organization (WHO). Drug abuse. Disponível em: <http://www.who.int/>

29. Silva CR. Caracterização do perfil dos usuários que interromperam o acompanhamento em um Centro de Atenção Psicossocial álcool e outras drogas (CAPSad). [tese] Salvador: Universidade Federal da Bahia; 2014. Disponível em: $<$ https:// repositorio.ufba.br/ri/bitstream/ri/15127/1/Carolina\%20 Rocha\%20Silva.pdf $>$.

30. Jesus RS, Sangoi RDS, Taschetto PL, De Brum TF, Piana M, Limberger JB. Perfil farmacoterapêutico de usuários de crack internados em hospital público de Santa Maria-RS. Disciplinarium Scientia. Série: Ciência da saúde 2014;15(1):3746.

31. Willhelm FF, Escobar M, Schweigert Perry IDS. Alterações na composição corporal e em parâmetros antropométricos de dependentes de crack internados em unidade de adição. J Bras Psiquiatr 2013;62(3):183-90. doi: http://dx.doi.org/10.1590/ S0047-20852013000300002

32. Recine E, Radaelli P. Obesidade e desnutrição. FS/ UnB, DAB/SPS/MS. Brasília: Ministério da Saúde; 2001. Disponível em: <http://www.turminha.mpf.mp.br/para-o-professor/para-oprofessor/publicacoes/Obesidadeedesnutricao.pdf $>$.

33. Santos FF, Magalhães LHVN, Sousa FAN, Marques CO, Torres MV, Leal SS. Analise da intervenção com realidade virtual e treino funcional na aptidão física de idosas. Revista Neurociências 2015;11(2):65-71. doi: http://dx.doi.org/10.5585/ ConsSaude.v14n1.5294

34. Júnior JBP. Efeito agudo da acupuntura, atividade lúdica e da realidade virtual sobre a dor após cirurgia cardíaca. [tese] São Cristovão: Universidade Federal de Sergipe; 2016. Disponível em: <https://ri.ufs.br/bitstream/riufs/4963/1/JOAO_ BARBOSA_PEREIRA_JUNIOR.pdf $>$.

35. Pelisser M, Garlipp DC, Sant'Anna MM. Correlação entre os resultados obtidos em testes motores e o desempenho em exergames em idosos. Cinergis 2017;18(3):179-84. doi: http:// dx.doi.org/10.17058/cinergis.v18i3.8851

36. Dias BB, Mota RS, Gênova TC, Tamborelli V, Pereira VV, Puccini PT. Aplicação da Escala de Equilíbrio de Berg para verificação do equilíbrio de idosos em diferentes fases do envelhecimento. RBCEH 2009;6(3):213-24. doi: http://dx.doi. org/10.5335/rbceh.2012.194

37. Wang C, Hsieh C, Olson SL, Wang C, Sheu C, Liang C. Psychometric Properties of the Berg Balance Scale in a Community-dwelling Elderly Resident Population in Taiwan. J Formos Med Assoc 2006;105(12):992-1000. doi: http://dx.doi. org/10.1016/S0929-6646(09)60283-7

38. Myiamoto ST, Lombardi JI, Berg KO, Ramos IR, Natour J. Braziliam version of the Berg Balance Scale. Braz J Med Biol Rev 2004;37(9):1411-21. doi: http://dx.doi.org/10.1590/S0100879X2004000900017

39. Yu J, Kim J. Effects of a physical activity program using exergame with elderly women. J Korean Acad Nurs 2015; 45(1):84-96. doi: http://dx.doi.org/10.4040/jkan.2015.45.1.84

40. Zaitsu K, Nishimura Y, Matsuguma H, Higuchi S. Association Between Extraversion and Exercise Performance Among Elderly Persons Receiving a Videogame Intervention. Games Health J 2015;4(5):375-95. doi: http://dx.doi.org/10.1089/g4h.2014.0119

Recebido em:14/02/2019 Aceito em:10/04/2019

Como citar: ROQUE BUENO, Lidiele; SERRÃO JUNIOR, Nelson Francisco; GRAUP, Susane. Efeito da realidade virtual no equilíbrio postural de usuários de um centro de atenção psicossocial álcool e drogas. Revista Interdisciplinar de Promoção da Saúde, Santa Cruz do Sul, v. 1, n. 4, dez. 2018. ISSN 2595-3664. Disponível em: <https://online.unisc.br/seer/index.php/ripsunisc/ article/view/13184>. Acesso em: 14 abr. 2019. doi:https://doi.org/10.17058/rips.v1i4.13184. 